\title{
Long-read genome sequencing identifies causal structural variation in a Mendelian disease
}

\author{
Jason D. Merker, MD, PhD ${ }^{1,2}$, Aaron M. Wenger, $\mathrm{PhD}^{3}$, Tam Sneddon, DPhil², \\ Megan Grove, MS, LCGC ${ }^{2}$, Zachary Zappala, PhD ${ }^{1,4}$, Laure Fresard, PhD ${ }^{1}$, Daryl Waggott, MSc ${ }^{5,6}$, \\ Sowmi Utiramerur, MS ${ }^{2}$, Yanli Hou, PhD ${ }^{1}$, Kevin S. Smith, PhD ${ }^{1}$, Stephen B. Montgomery, PhD 1,4, \\ Matthew Wheeler, MD, PhD ${ }^{5,6}$, Jillian G. Buchan, PhD ${ }^{1,2}$, Christine C. Lambert, BA ${ }^{3}$, Kevin S. Eng, MS $^{3}$, \\ Luke Hickey, $\mathrm{BS}^{3}$, Jonas Korlach, $\mathrm{PhD}^{3}$, James Ford, $\mathrm{MD}^{4,5,7}$ and Euan A. Ashley, MRCP, DPhil ${ }^{2,4,5,6}$
}

Purpose: Current clinical genomics assays primarily utilize short-read sequencing (SRS), but SRS has limited ability to evaluate repetitive regions and structural variants. Long-read sequencing (LRS) has complementary strengths, and we aimed to determine whether LRS could offer a means to identify overlooked genetic variation in patients undiagnosed by SRS.

Methods: We performed low-coverage genome LRS to identify structural variants in a patient who presented with multiple neoplasia and cardiac myxomata, in whom the results of targeted clinical testing and genome SRS were negative.

Results: This LRS approach yielded 6,971 deletions and 6,821 insertions $>50 \mathrm{bp}$. Filtering for variants that are absent in an unrelated control and overlap a disease gene coding exon identified three deletions and three insertions. One of these, a heterozygous
2,184 bp deletion, overlaps the first coding exon of PRKAR1A, which is implicated in autosomal dominant Carney complex. RNA sequencing demonstrated decreased PRKAR1A expression. The deletion was classified as pathogenic based on guidelines for interpretation of sequence variants.

Conclusion: This first successful application of genome LRS to identify a pathogenic variant in a patient suggests that LRS has significant potential for the identification of disease-causing structural variation. Larger studies will ultimately be required to evaluate the potential clinical utility of LRS.

Genet Med advance online publication 22 June 2017

Key Words: Carney complex; long-read sequencing; PacBio; PRKAR1A; structural variant

\section{INTRODUCTION}

Short-read sequencing (SRS) methods are primarily used in clinical laboratory medicine because of their cost-effectiveness and low per-base error rate. However, these methods do not capture the full range of genomic variation. ${ }^{1}$ Areas of low complexity, such as repeats, and areas of high polymorphism, such as the human leukocyte antigen region, present challenges to SRS and reference-based genome assembly. Indeed, with 100 base pair (bp) read length, fully $5 \%$ of the genome cannot be uniquely mapped. ${ }^{2}$ In addition, many diseases are caused by repeats in a range beyond the resolution of SRS. Another challenge comes in the form of structural variation, and although SRS has been very successful in the discovery of single-nucleotide and small insertion-deletion variation, recent findings suggest we have greatly underestimated the extent and complexity of structural variation in the genome. ${ }^{3,4}$

Long-read sequencing (LRS), typified by PacBio singlemolecule, real-time (SMRT) sequencing, offers complementary strengths to those of SRS. PacBio LRS produces reads of several thousand base pairs with uniform coverage across sequence contexts. ${ }^{5}$ Individual long reads have a lower accuracy $(85 \%)$ than short reads, but errors are random and are correctable with sufficient coverage, leading to high consensus accuracy. ${ }^{5,6}$ Furthermore, long reads are more accurately mapped to the genome and access regions that are beyond the reach of short reads. ${ }^{1}$ Of note, recent PacBio LRS de novo human genome assemblies have revealed tens of thousands of structural variants per genome, many times more than previously observed with SRS. ${ }^{3,7}$ These capabilities, together with continuing progress in throughput and cost, may make LRS an option for broader application in human genomics.

Here, we report the use of low-coverage genome LRS to secure a diagnosis of Carney complex where clinical singlegene testing and genome SRS had been unsuccessful. This initial application of LRS to identify a pathogenic structural variant in a patient, when considered alongside other prior

\footnotetext{
${ }^{1}$ Department of Pathology, Stanford University, Stanford, California, USA; ${ }^{2}$ Stanford Medicine Clinical Genomics Service, Stanford Health Care, Stanford, California, USA; ${ }^{3}$ Pacific Biosciences, Menlo Park, California, USA; ${ }^{4}$ Department of Genetics, Stanford University, Stanford, California, USA; ${ }^{5}$ Department of Medicine, Stanford University, Stanford, California, USA; ${ }^{6}$ Stanford Center for Inherited Cardiovascular Disease, Stanford University, Stanford, California, USA; ${ }^{7}$ Stanford Cancer Institute, Stanford, California, USA. Correspondence: Euan A. Ashley (euan@stanford.edu)

The first two authors contributed equally to this work. 
studies, suggests that LRS can identify disease-causing structural variants that are difficult to detect using current technologies. Larger studies are needed to evaluate the molecular diagnostic yield and potential clinical utility of LRS.

\section{MATERIALS AND METHODS \\ Case report}

The patient is an Asian/Hispanic male, the product of an uncomplicated term pregnancy who was hospitalized for the first 10 days of life for cardiac and respiratory issues (Figure 1a). He remained well until the age of 7 years, when, following the discovery of a heart murmur, he was found to have a left atrial myxoma that was surgically removed. At 10 years, he was noted to have a testicular mass that, at orchiectomy, was found to be a Sertoli-Leydig cell tumor. At 13 years, he was found to have a pituitary tumor and initial conservative management was adopted. Aged 16, he was noted to have both an adrenal microadenoma and recurrence of the cardiac myxomata in the left ventricle and right atrium. Blue nevi were reported. He underwent a second surgical resection of the myxomata with uncomplicated recovery. When he was 18, recurrent cardiac myxomata, including a right ventricular and two left ventricular tumors, were once again resected and a Gore-Tex patch was placed in the right ventricular wall. In the immediate post-operative period, he suffered ventricular tachycardia (VT) and cardiac arrest with spontaneous return of circulation. At this time, a genetics evaluation suggested the possibility of Carney complex, but clinical sequencing of PRKAR1A was negative for disease-causing variation. At age 19, after multiple thyroid nodules were noted on ultrasound, he was diagnosed with ACTH-independent Cushing's syndrome, secondary to the adrenal microadenoma. At 21, he was found to have a pituitary lesion and acromegaly. He subsequently underwent trans-sphenoidal resection of the pituitary tumor with pathology confirming a growth-hormone producing pituitary adenoma. At this time, he was found to have recurrent myxomata in the left ventricular outflow tract, which have subsequently increased in size (Figure $\mathbf{1 b}-\mathbf{c}$ ). To date, these have been treated conservatively with anticoagulation to reduce the risk of stroke. As of 2016, he is under consideration for heart transplantation, and the transplant team judged molecular confirmation of the clinical diagnosis desirable prior to transplant listing.

\section{Short read genome sequencing and analysis}

A library was generated from genomic DNA using the Illumina TruSeq DNA PCR-Free Library Prep Kit (Illumina, San Diego, CA) and sequencing was performed on the Illumina HiSeq 2500 System with paired-end 100 bp reads to a 36-fold mean depth of coverage. The Stanford Medicine Clinical Genomics Service performed the data analysis and variant curation. Single-nucleotide variants and small insertions and deletions were identified using MedGAP v2.0, a pipeline based on GATK best practices for data preprocessing and variant discovery with GATK HaplotypeCaller v3.1.1. ${ }^{8}$ This analysis pipeline did not identify any variants that would explain the clinical findings in the patient. Multiple short-read structural variant callers, including Pindel, Lumpy, BreakDancer, Manta, CNVKit, and CNVnator, were retrospectively used to identify structural variants, ${ }^{9-14}$ as described in the Supplementary Materials and Methods online.

\section{Long read genome sequencing and analysis}

Following informed consent under a protocol approved by the Stanford University Institutional Review Board, low-coverage
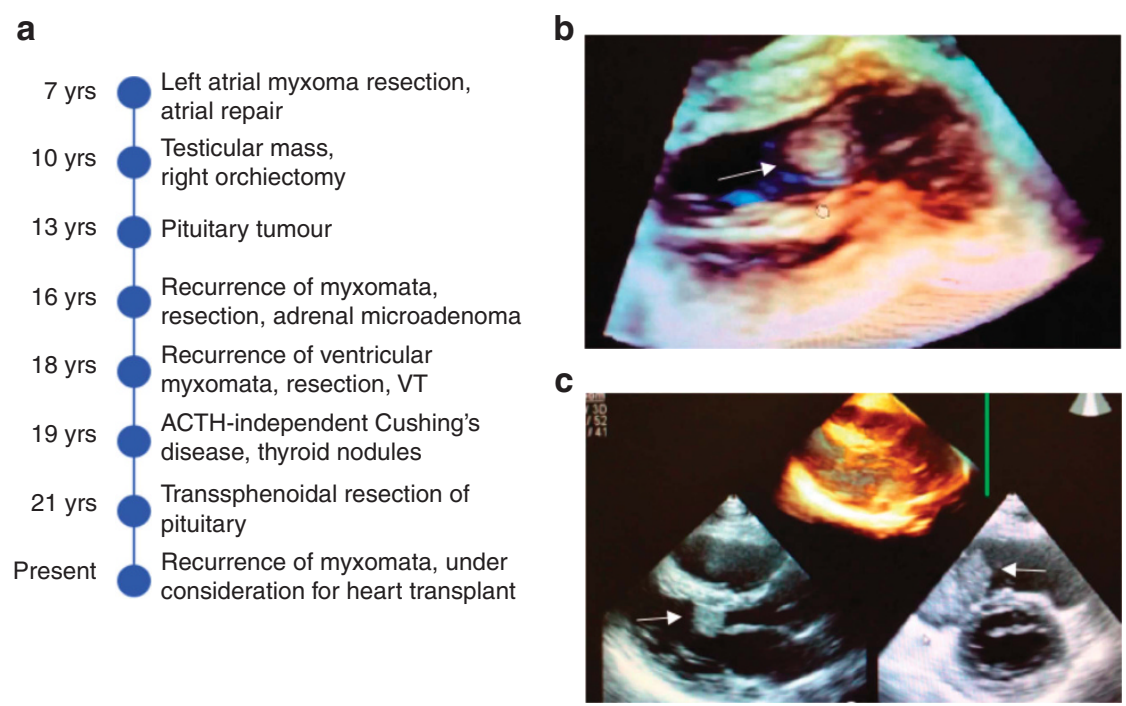

Figure 1 Clinical history and three-dimensional transthoracic echocardiography of patient with multiple neoplasia including cardiac myxomata. (a) Patient narrative. (b) A $2 \times 3 \mathrm{~cm}$ myxoma is seen in the left ventricular outflow tract (white arrow). (c) The $2 \times 3 \mathrm{~cm}$ myxoma is seen from another perspective (lower left, white arrow). A $5 \times 4 \mathrm{~cm}$ myxoma is seen in the right atrium (lower right, white arrow). VT, ventricular tachycardia. 
genome LRS was performed on the PacBio Sequel System (Pacific Biosciences of California, Menlo Park, CA) to evaluate structural variation. The sequencing generated a ninefold mean depth of coverage with an average read length of $>9 \mathrm{~kb}$. Further details are provided in the Supplementary Materials and Methods.

\section{Other methods}

RNA sequencing and parentage studies are described in Supplementary Materials and Methods.

\section{RESULTS}

The resulting call set from LRS consisted of 6,971 deletions and 6,821 insertions $>50 \mathrm{bp}$ (Supplementary Table S1). To prioritize candidate pathogenic variants, the call set was filtered to exclude variants within a segmental duplication or present in the unrelated control individual NA12878. This left 2,476 deletions and 3,171 insertions. Focusing on variants that overlap a RefSeq coding exon resulted in 39 deletions and 16 insertions, with 3 deletions and 3 insertions in genes linked to a genetic disease in OMIM. The three OMIM genes, as well as phenotype and mode of inheritance, included in deletions are CASP8 (autoimmune lymphoproliferative syndrome type IIB, autosomal recessive), CD209 (susceptibility to or protection from certain pathogens), and PRKAR1A (Carney complex, autosomal dominant); and the three OMIM genes included in insertions are: KALRN (susceptibility to coronary heart disease), PAPSS2 (brachyolmia, autosomal recessive), and PCDH15 (Usher syndrome, autosomal recessive). Manual review of the six candidate variants and correlation with phenotype identified a heterozygous deletion that removes the first coding exon of PRKAR1A (NM_212472.2). Germ-line variants in PRKAR1A cause Carney complex, type 1 (MIM 160980), an autosomal dominant multiple neoplasia syndrome.

Two of four reads at the locus unambiguously support the presence of a deletion (Figure 2a). Because of the random errors in LRS, individual reads from the same allele can have slight disagreements, and two reads can be insufficient to define exact deletion breakpoints with full confidence. Here, the higher-quality read supports a $2,184 \mathrm{bp}$ deletion of GRCh37/hg19 chr17:66,510,475-66,512,658 (NC_000017.10: g.66510475_66512658del). This heterozygous deletion variant was validated by Sanger sequencing, confirming the precise breakpoints identified by LRS (Figure 2 b). Sanger sequencing of the parental specimens did not detect this deletion, and single-nucleotide variant-based identity testing was consistent with both parental samples being from the biological parents of the proband, indicating a de novo variant.

RNA sequencing of peripheral blood mononuclear cells from the proband demonstrates that the observed genomic deletion has an effect at the RNA level. The overall PRKAR1A expression level in the proband is significantly lower than in equivalently processed controls (Supplementary Figure S1A). When relative expression is examined at the exon level, exon 2 , which is deleted in the genomic DNA, demonstrates the largest observed reduction, but 10 of 11 exons demonstrate a trend toward reduced expression (Supplementary Figure S1B). Splicing analysis identifies an isoform that skips exon 2 in the proband that is not detected in any of the controls (Supplementary Figure S1C). Overall, this splice isoform in the proband that skips exon 2 is observed at an approximately fourfold lower level than that of the canonical isoform. The

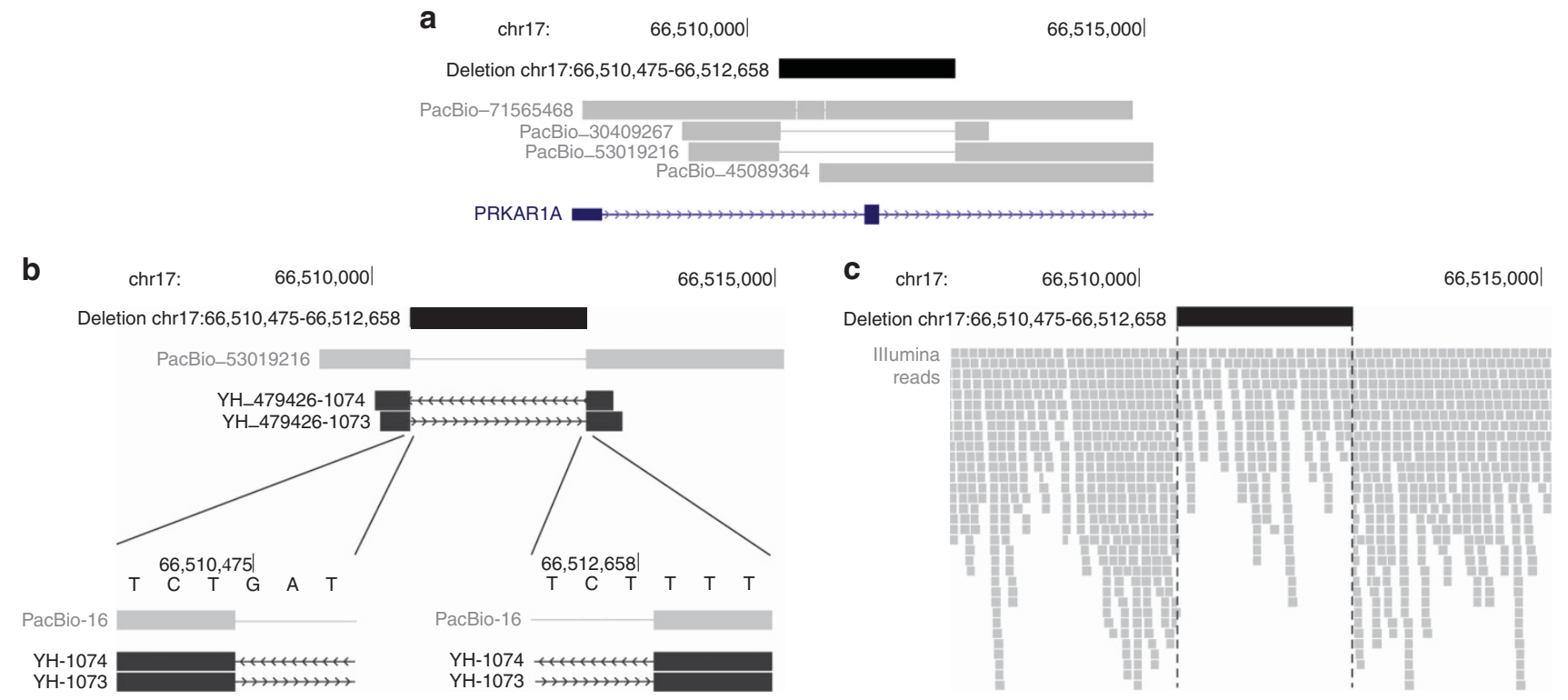

Figure 2 Heterozygous deletion in PRKAR1A. (a) PacBio long reads identify a heterozygous 2,184 bp deletion that includes the first coding exon of PRKAR1A. Two of four reads at the locus support the deletion. (b) Sanger sequencing confirms the deletion. The forward (YH_479426-1073) and reverse (YH_479426-1074) sequences from a representative amplicon agree to the base pair with the higher quality PacBio read, PacBio_53019216. (c) Illumina short reads support the heterozygous deletion variant through a drop in read coverage and clipped reads at the deletion breakpoints. 
genomic DNA encoding the transcribed exons of PRKAR1A did not contain any heterozygous sites, so we were unable to analyze allele specific expression.

Using the ACMG Standards and Guidelines for the interpretation of sequence variants, ${ }^{15}$ this variant was categorized as pathogenic based on: (i) identification of a null variant in a gene where loss of function is a known mechanism of disease (PVS1), and (ii) de novo variant in a patient with disease and no family history (where both maternity and paternity confirmed, PS2).

It is difficult to call structural variants in SRS data with simultaneously high sensitivity and the specificity necessary for clinical laboratory testing. Nevertheless, once a small candidate gene list or approximate breakpoints are known, many variants can be identified retrospectively. ${ }^{5}$ In such cases, SRS often provides exact breakpoints to refine the variant discovered by LRS. ${ }^{16}$ Manual inspection of SRS data from the PRKAR1A locus shows support for the heterozygous deletion through a drop in read depth and alignment clipping at the deletion breakpoints (Figure 2c). Multiple short-read structural variant callers, including Pindel, Lumpy, BreakDancer, Manta, CNVKit, and CNVnator, were retrospectively used to identify structural variants. ${ }^{9-14}$ Pindel, Lumpy, BreakDancer, and Manta all identify a deletion in the locus. Pindel and Manta approximate the breakpoints identified from LRS and Sanger sequencing. Comparisons of the variant filtering results and overlap for LRS and SRS for Pindel and Manta are provided in Supplementary Tables S1 and S2.

\section{DISCUSSION}

Carney complex is a rare, autosomal dominant disease diagnosed using clinical criteria, including pigmented skin abnormalities, myxomas, endocrine tumors and dysfunction, and schwannomas. ${ }^{17}$ Two or more major diagnostic criteria are required for a definitive diagnosis of Carney complex ${ }^{18}$, and this patient meets three: (i) cardiac myxomas, (ii) largecell calcifying Sertoli cell tumor, and (iii) acromegaly as a result of a growth hormone-producing pituitary adenoma (all histologically confirmed). Additional signs suggestive of Carney complex include skin findings (a few lentigines and multiple blue nevi) and multiple thyroid nodules detected by ultrasound in an individual older than 18 years. Genome and RNA sequencing identified a de novo pathogenic deletion in PRKAR1A, providing molecular confirmation of the diagnosis.

This case demonstrates the ability of genome LRS to effect the detection of causal structural variation in a rare disease, and to our knowledge, this is the first reported application of genome LRS to identify a pathogenic variant in a patient. Although this 2,184 bp deletion can be identified through manual inspection of the aligned read data and short-read structural variant callers, these approaches are not practical for genome-wide application, owing to limited throughput and high false-positive call rates, respectively. In the future, clinical-grade genomics would ideally provide strong precision and recall across the full spectrum of genetic variation.
SRS has decreased sensitivity for insertion and deletion variant detection as the size of the event increases, and it can miss up to $80 \%$ of the structural variants in an individual genome. $^{3}$ Current cytogenomic arrays have a maximum resolution > 5-10 kb. ${ }^{19}$ This leaves an opening for a technology that can detect insertions and deletions too large for SRS, e.g., > $50 \mathrm{bp}$, and too small for cytogenomic arrays. LRS appears to be capable of identifying much of the missed variation, and manifests high recall of structural variants even at low depths of coverage. ${ }^{16}$ This initial proof-of-concept case demonstrates that this variation can be clinically relevant. We suggest that larger studies on the molecular diagnostic yield of LRS will be required to fully evaluate the relative performance of LRS versus SRS for the identification of intermediate size insertions and deletions and to determine the ultimate clinical utility of this approach. Likewise, cost reductions in LRS technologies will be required prior to any clinical implementation.

In the current manuscript, we describe the use of long-read genome sequencing to identify a $\sim 2.2 \mathrm{~kb}$ deletion in PRKAR1A in a patient with Carney complex, providing a molecular explanation for disease. This first successful application of genome LRS to identify a pathogenic variant in a patient, when considered in the context of prior studies, suggests that LRS may be one approach to the identification of disease-causing structural variants that are difficult to detect using current technologies.

\section{SUPPLEMENTARY MATERIAL}

Supplementary material is linked to the online version of the paper at http://www.nature.com/gim

\section{ACKNOWLEDGMENTS}

The authors thank the research subject and clinical care teams for their participation in this research study; Chen-Shan (Jason) Chin for helpful discussions; and Primo Baybayan and Matt Boitano for PacBio library preparation and sequencing.

\section{DISCLOSURE}

A.M.W., C.C.L., K.S.E., L.H., and J.K. are employees and shareholders of Pacific Biosciences, a company commercializing DNA sequencing technologies. The other authors declare no conflict of interest.

\section{REFERENCES}

1. Ashley EA. Towards precision medicine. Nat Rev Genet. 2016;17: 507-522.

2. Goldfeder RL, Priest JR, Zook JM, et al. Medical implications of technical accuracy in genome sequencing. Genome Med. 2016;8:24.

3. Chaisson MJ, Huddleston J, Dennis MY, et al. Resolving the complexity of the human genome using single-molecule sequencing. Nature 2015;517: 608-611.

4. Huddleston J, Chaisson MJ, Meltz Steinberg K, et al. Discovery and genotyping of structural variation from long-read haploid genome sequence data. Genome Res 2017;27:677-685.

5. Chaisson MJ, Wilson RK, Eichler EE. Genetic variation and the de novo assembly of human genomes. Nat Rev Genet 2015;16:627-640.

6. Chin CS, Alexander DH, Marks P, et al. Nonhybrid, finished microbial genome assemblies from long-read SMRT sequencing data. Nat Methods 2013;10:563-569. 
7. Seo JS, Rhie A, Kim J, et al. De novo assembly and phasing of a Korean human genome. Nature 2016;538:243-247.

8. Van der Auwera GA, Carneiro MO, Hartl C, et al. From FastQ data to high confidence variant calls: the Genome Analysis Toolkit best practices pipeline. Curr Protoc Bioinformatics 2013:43:11.10.11-33.

9. Ye K, Schulz MH, Long Q, Apweiler R, Ning Z. Pindel: a pattern growth approach to detect break points of large deletions and medium sized insertions from paired-end short reads. Bioinformatics 2009;25: 2865-2871.

10. Layer RM, Chiang C, Quinlan AR, Hall IM. LUMPY: A probabilistic framework for structural variant discovery. Genome Biol 2014;15:R84.

11. Chen K, Wallis JW, McLellan MD, et al. BreakDancer: an algorithm for high-resolution mapping of genomic structural variation. Nat Methods 2009;6:677-681.

12. Chen $X$, Schulz-Trieglaff $O$, Shaw $R$, et al. Manta: rapid detection of structural variants and indels for germline and cancer sequencing applications. Bioinformatics 2016;32:1220-1222.

13. Talevich E, Shain AH, Botton T, Bastian BC. CNVkit: genome-wide copy number detection and visualization from targeted DNA sequencing. PLOS Comput Biol. 2016;12:e1004873.
14. Abyzov A, Urban AE, Snyder M, Gerstein M. CNVnator: an approach to discover, genotype, and characterize typical and atypical CNVs from family and population genome sequencing. Genome Res 2011;21: 974-984.

15. Richards S, Aziz N, Bale $S$, et al. Standards and guidelines for the interpretation of sequence variants: a joint consensus recommendation of the American College of Medical Genetics and Genomics and the Association for Molecular Pathology. Genet Med 2015;17:405-424

16. English AC, Salerno WJ, Hampton OA, et al. Assessing structural variation in a personal genome-towards a human reference diploid genome. BMC Genomics 2015;16:286.

17. Stratakis CA, Salpea P, Raygada M. Carney Complex. In: Pagon RA, Adam MP, Ardinger $\mathrm{HH}$, et al. (eds). GeneReviews. Seattle, WA: University of Washington, 1993-2017.

18. Mateus C, Palangie A, Franck N, et al. Heterogeneity of skin manifestations in patients with Carney complex. I Am Acad Dermatol. 2008;59:801-810.

19. Le Scouarnec S, Gribble SM. Characterising chromosome rearrangements: recent technical advances in molecular cytogenetics. Heredity 2012;108:75-85. 